Supporting Information

\title{
Stability tuning of vapor-adsorbed state of vapochromic Pt(II) complex by introduction of chiral moiety
}

\author{
Yasuhiro Shigeta, Atsushi Kobayashi,* Masaki Yoshida, and Masako Kato* \\ Department of Chemistry, Faculty of Science, Hokkaido University, North 10 West 8, Kita-ku, \\ Sapporo, Hokkaido 060-0810
}

\section{Contents}

Figure S1. ${ }^{1} \mathrm{H}$ NMR signals of R-1, S-1 and rac-1 with chiral shift reagent.

Figure S2. PXRD patterns of as-synthesized R-1 and S-1.

Figure S3. Thermogravimetric analyses of anhydrous R-1 and rac-1.

Figure S4. Thermogravimetric analysis for benzene-vapor-exposed R-1 and rac-1.

Figure S5. Experimental PXRD patterns of R-1 and rac-1 after toluene or benzene vapor exposure.

Figure S6. PXRD pattern changes of rac-1 upon toluene vapor exposure and drying.

Figure S7. PXRD patterns of R-1 after exposure to several chiral vapors.

Figure S8. Absorption and diffuse reflectance spectra of R-1 and rac-1.

Figure S9. Photographs of R-1 • toluene and rac-1 •toluene after vacuum treatment for $4 \mathrm{~h}$.

Table S1. Selected bond lengths for R-1 and rac-1 crystal at $150 \mathrm{~K}$. 


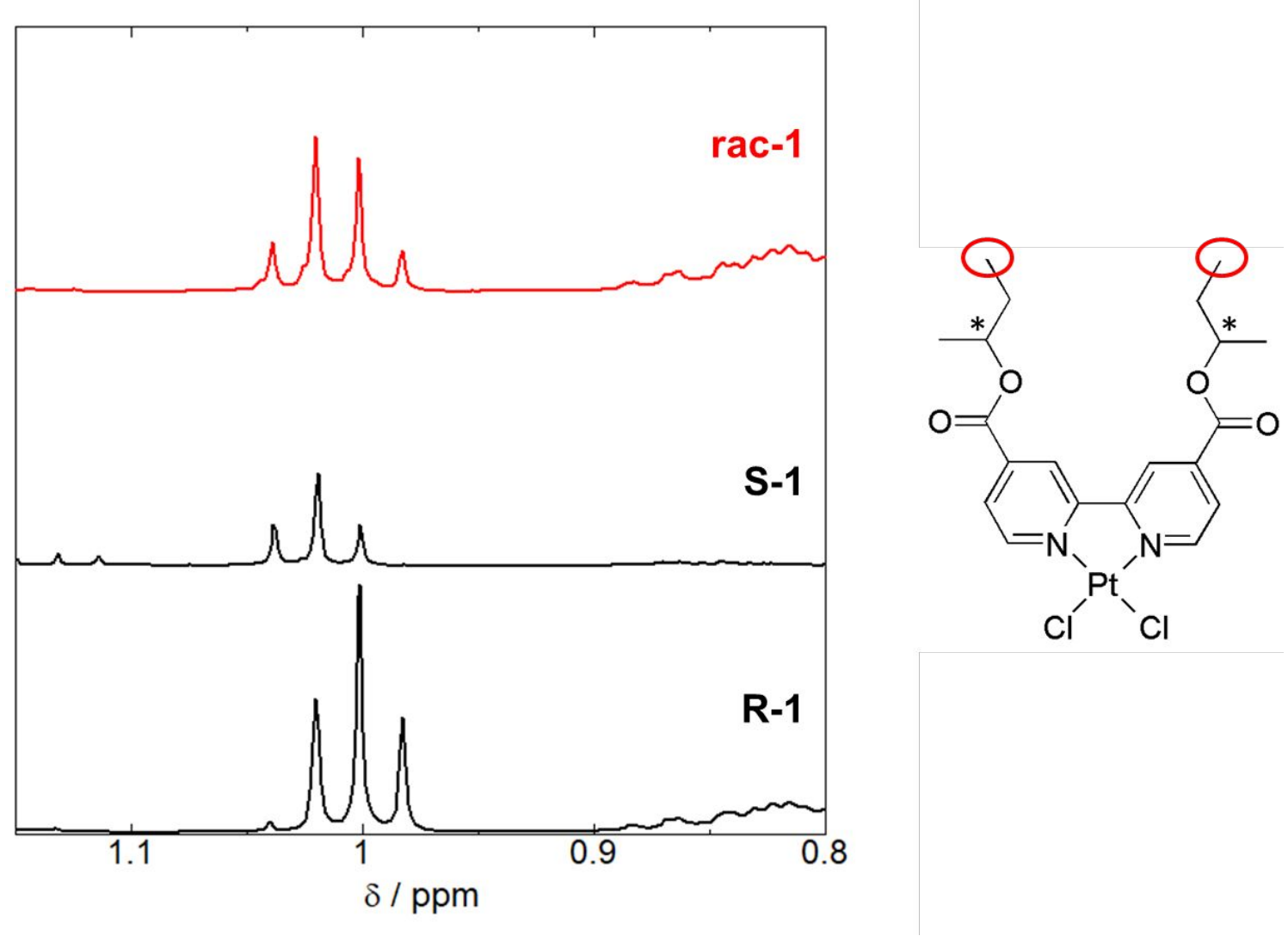

Figure S1. ${ }^{1}$ H NMR signals of R-1 (bottom), S-1(middle) and rac-1(top) of the terminal methyl proton of the butyl ester chain which indicated as red circles with chiral shift reagent (Chirabite-AR, purchased from TCI). 


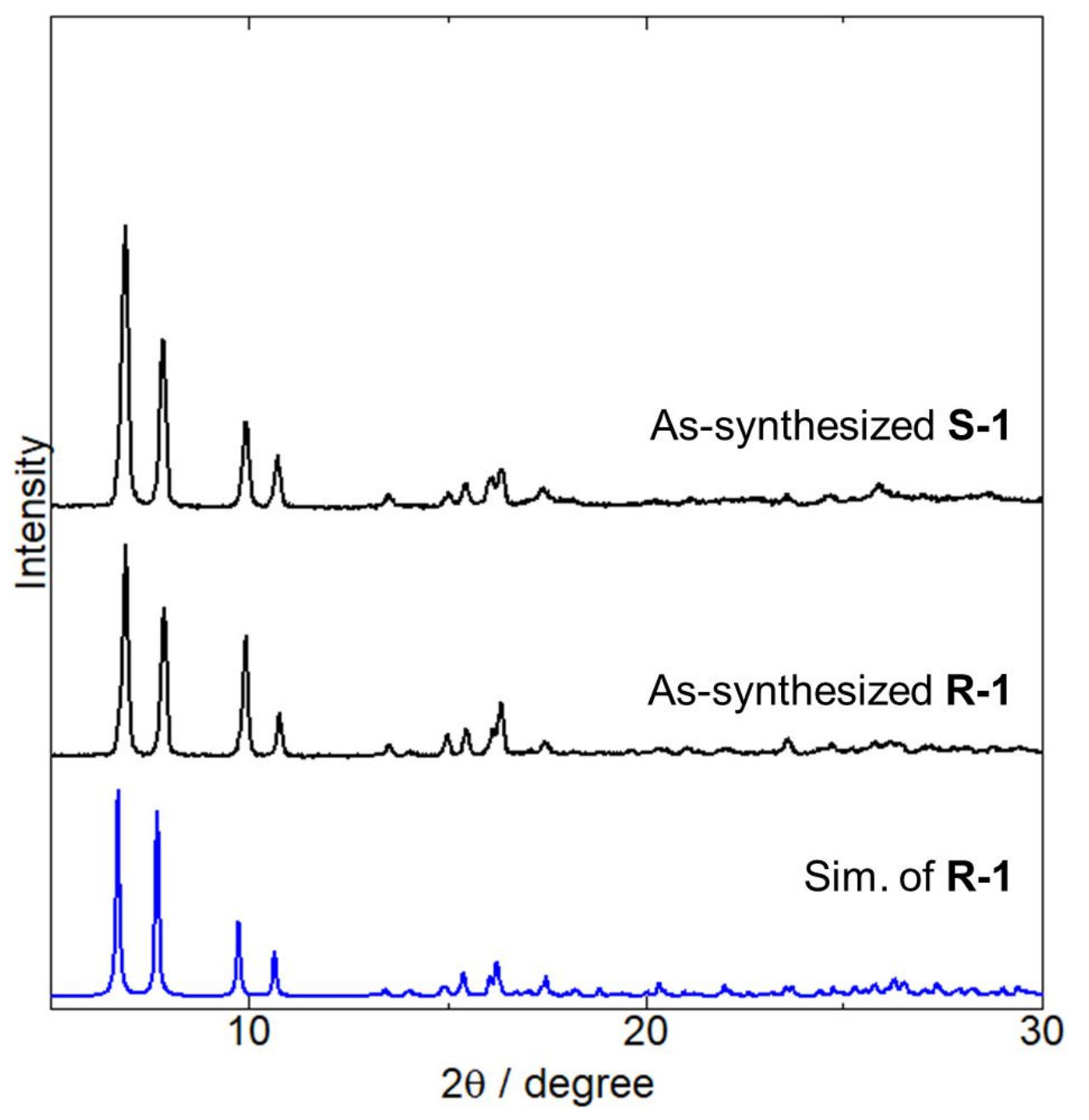

Figure S2. PXRD patterns of S-1 (top) and R-1 (middle) compared with the simulation pattern of R-1(bottom). 


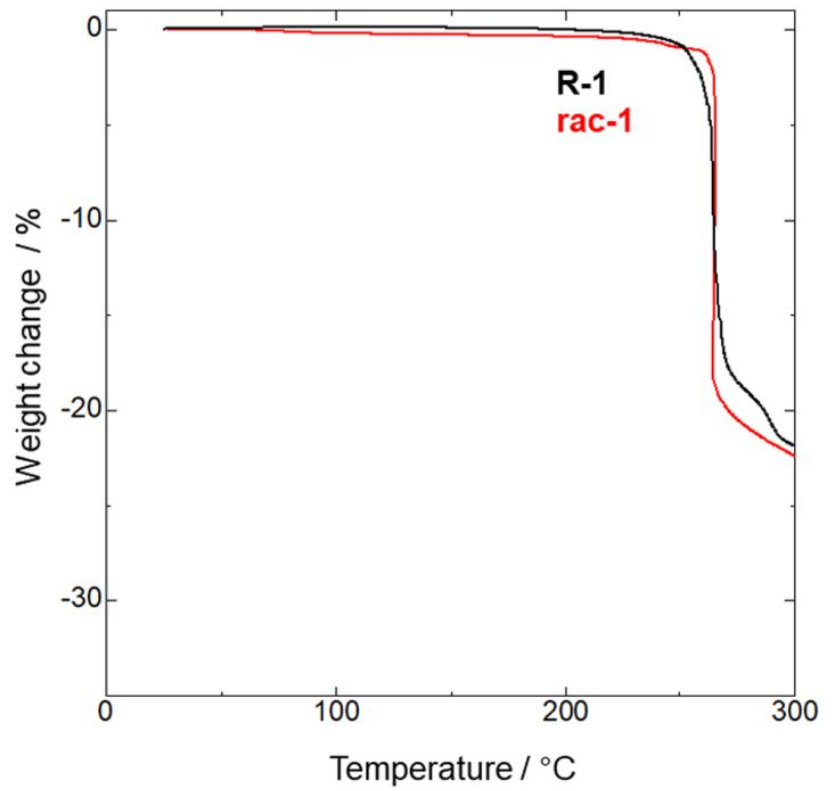

Figure S3. Thermogravimetric analysis of R-1(black) and rac-1(red) in Ar flow (0.3 L/min).

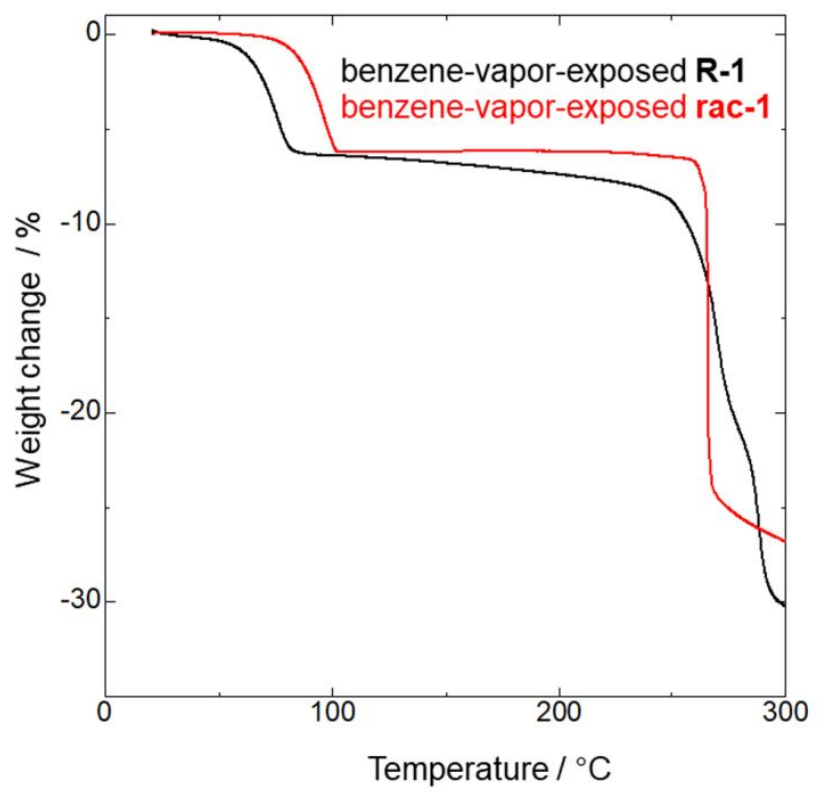

Figure S4. Thermogravimetric analysis of benzene-vapor-exposed R-1(black) and rac-1(red) in Ar flow $(0.3 \mathrm{~L} / \mathrm{min})$. 


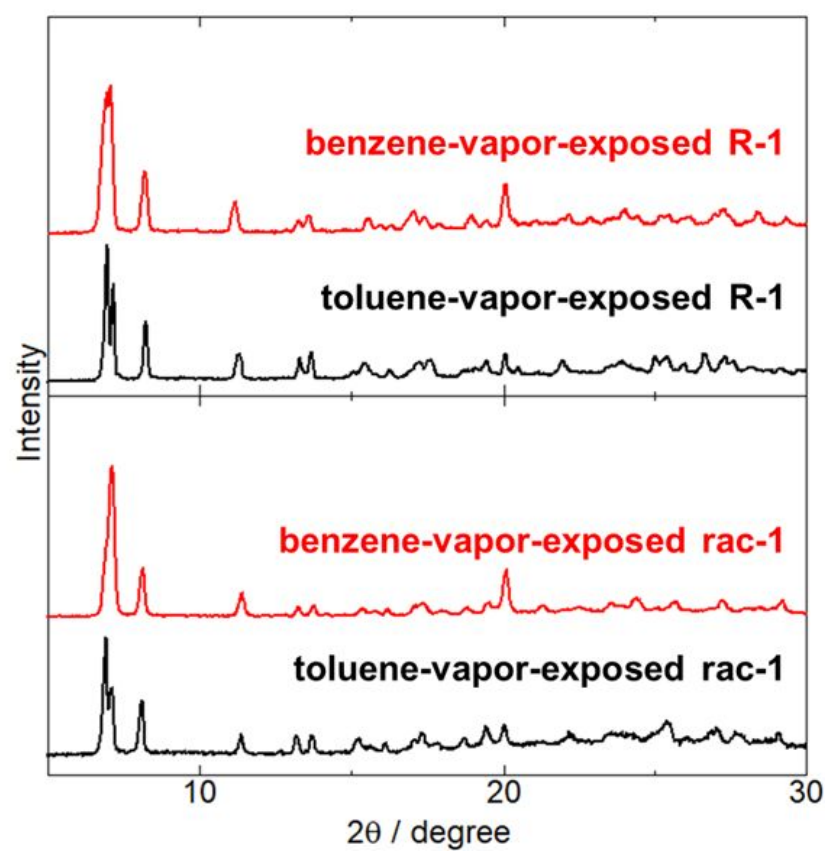

Figure S5. Experimental PXRD patterns of R-1 (top) and rac-1 (bottom) after toluene (black) or benzene (red) vapor exposure for 3 days at $303 \mathrm{~K}$. 


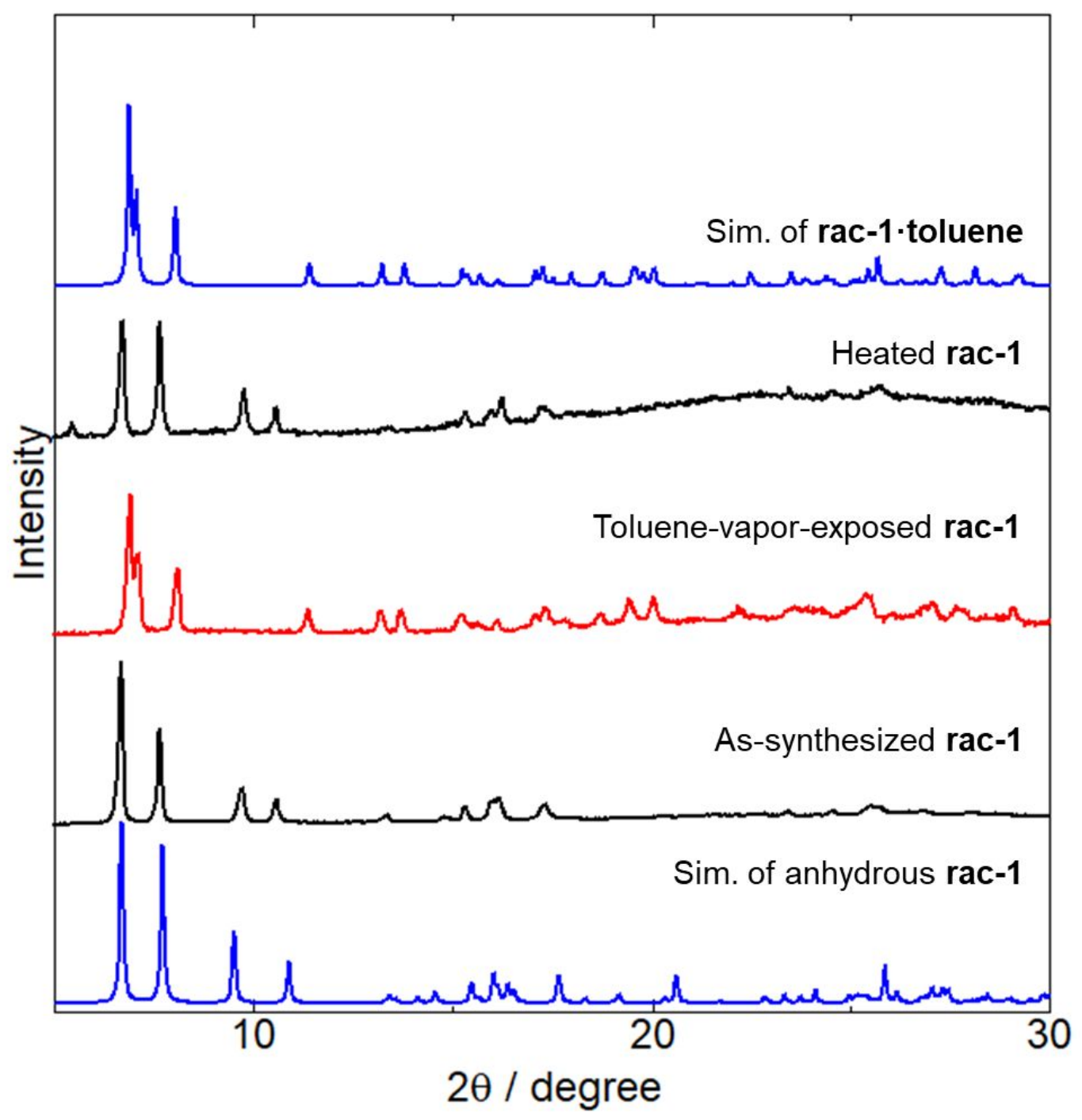

Figure S6. PXRD pattern changes of rac-1 upon exposure to toluene vapor at $30{ }^{\circ} \mathrm{C}$ for 5 days. The toluene-exposed samples were dried under vacuum for 1day and subsequent heating at $120^{\circ} \mathrm{C}$ for 2 hours. 


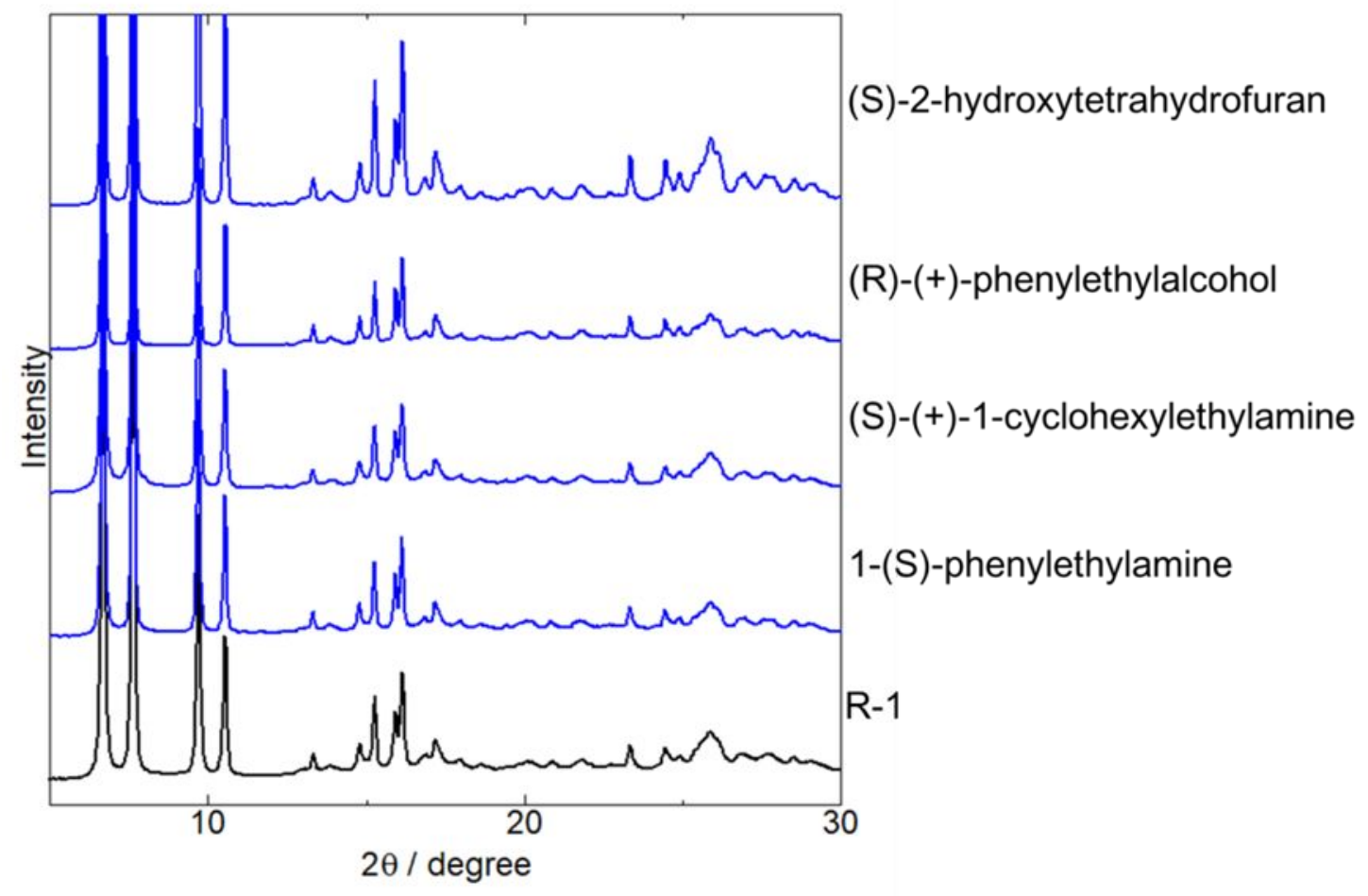

Figure S7. PXRD patterns of R-1 after exposure to several chiral vapors. 


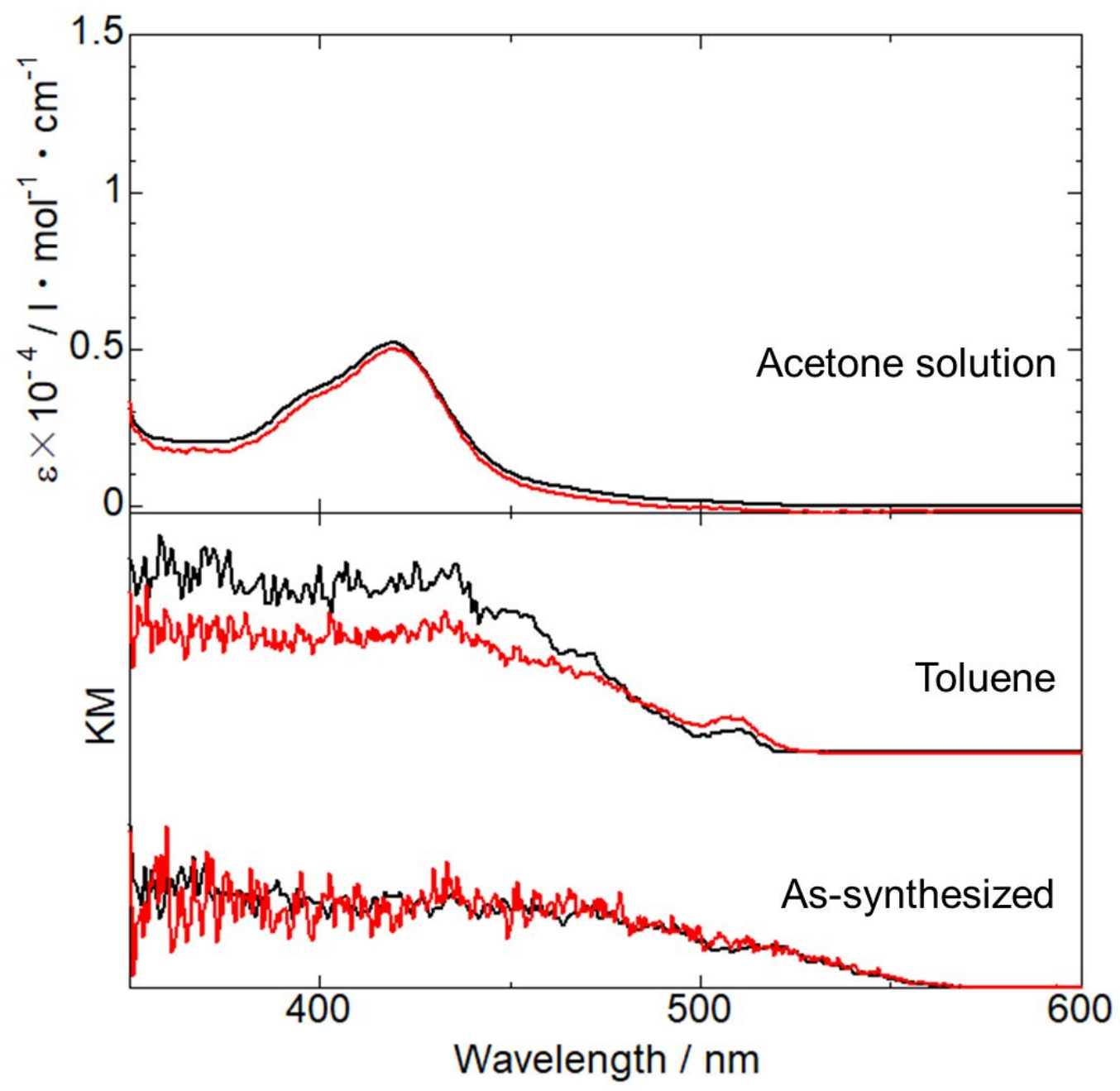

Figure S8. UV-Vis absorption spectra in the acetone solutions (top) and diffuse reflectance spectra in the solid state (bottom) of R-1 (black lines) and rac-1 (red lines). 
R-1

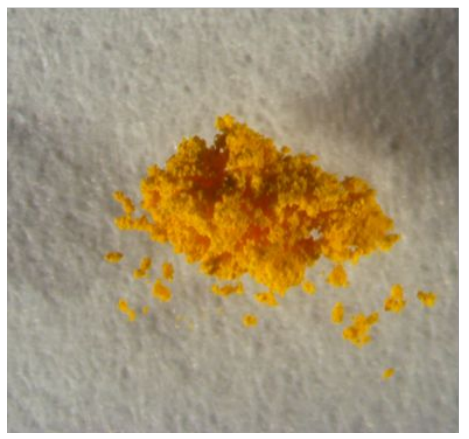

Figure S9. Photographs of R-1 •toluene and rac-1 • toluene after vacuum treatment for $4 \mathrm{~h}$.

\section{rac-1}

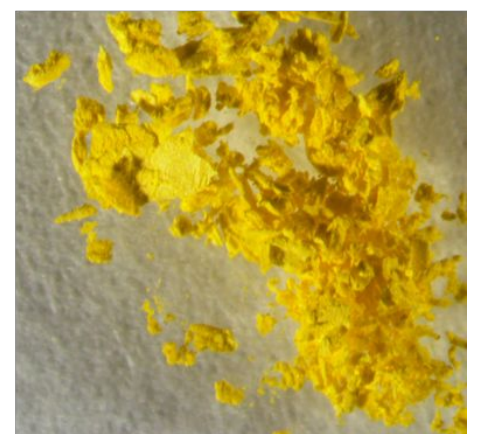


Table S1. The Pt-N and Pt-Cl bond lengths of R-1 and rac-1 crystals at $150 \mathrm{~K}$.

\begin{tabular}{|c|c|c|}
\hline Complex & R-1 & Rac-1 \\
\hline Pt1-N1 & $2.02(1) \AA$ & $2.016(7) \AA$ \\
\hline $\mathrm{Pt} 1-\mathrm{N} 2$ & $2.03(1) \AA$ & $2.016(5) \AA$ \\
\hline Pt1-Cl1 & $2.293(5) \AA$ & $2.293(2) \AA$ \\
\hline $\mathrm{Pt} 1-\mathrm{Cl} 2$ & $2.290(4) \AA$ & $2.300(2) \AA$ \\
\hline $\mathrm{Pt} 2-\mathrm{N} 3$ & $2.02(1) \AA$ & - \\
\hline $\mathrm{Pt} 2-\mathrm{N} 4$ & $2.01(1) \AA$ & - \\
\hline $\mathrm{Pt} 2-\mathrm{Cl} 3$ & $2.292(4) \AA$ & - \\
\hline $\mathrm{Pt} 2-\mathrm{Cl} 4$ & $2.296(5) \AA$ & - \\
\hline Pt3-N5 & $1.98(1) \AA$ & - \\
\hline $\mathrm{Pt3}-\mathrm{N} 6$ & $2.01(2) \AA$ & - \\
\hline $\mathrm{Pt} 3-\mathrm{Cl} 5$ & $2.289(6) \AA$ & - \\
\hline $\mathrm{Pt3}-\mathrm{Cl} 6$ & $2.297(6) \AA$ & - \\
\hline Pt4-N7 & $2.00(1) \AA$ & - \\
\hline Pt4-N8 & $2.02(1) \AA$ & - \\
\hline $\mathrm{Pt} 4-\mathrm{Cl} 7$ & $2.293(6) \AA$ & - \\
\hline $\mathrm{Pt} 4-\mathrm{Cl} 8$ & $2.289(4) \AA$ & - \\
\hline Pt5-N9 & $2.01(1) \AA$ & - \\
\hline Pt5-N10 & $2.01(2) \AA$ & - \\
\hline Pt5-C19 & $2.294(6) \AA$ & - \\
\hline Pt5-C110 & $2.299(4) \AA$ & - \\
\hline Pt6-N11 & $2.04(2) \AA$ & - \\
\hline Pt6-N12 & $2.02(1) \AA$ & - \\
\hline Pt6-C111 & $2.293(4) \AA$ & - \\
\hline Pt6-C112 & $2.293(5) \AA$ & - \\
\hline
\end{tabular}

